\title{
Treatment Failure After Rotational Atherectomy and Balloon Angioplasty in Recurrent Cystic Adventitial Disease of the Popliteal Artery: a Case Report
}

\section{Jin Woo Yoon}

Chung-Ang University Hospital: Chung Ang University Hospital

Woosun Choi ( $\square$ radiochoi527@gmail.com)

Chung-Ang University Hospital: Chung Ang University Hospital https://orcid.org/0000-0002-4874-3781

\section{Suh Min Kim}

Chung-Ang University Hospital: Chung Ang University Hospital

Joonho Hur

Chung-Ang University Hospital: Chung Ang University Hospital

\section{Research Article}

Keywords: Cystic adventitial disease, Recurrent cystic adventitial disease, Rotational atherectomy, Balloon angioplasty

Posted Date: September 1st, 2021

DOl: https://doi.org/10.21203/rs.3.rs-832436/v1

License: (9) This work is licensed under a Creative Commons Attribution 4.0 International License. Read Full License

Version of Record: A version of this preprint was published at Journal of International Medical Research on March 1st, 2022. See the published version at https://doi.org/10.1177/03000605221086149. 


\section{Abstract}

Background: Cystic adventitial disease (CAD) is a rare non-atherosclerotic condition in which fluid accumulates in the sub-adventitial layer, compressing the lumen of the arteries and veins. Historically, surgical treatment of CAD is preferred over endovascular or minimally invasive techniques owing to the considerably lower recurrence rates after surgery.

Case presentation: We here report the first case in which rotational atherectomy was performed using Jetstream XC (Boston Scientific, Natick, MA) for recurrent CAD. Post-procedure angiography showed that satisfactory increase in the luminal diameter. However, the patient's symptoms recurred 1 day after procedure, and repeat Computed tomography angiography (CTA) showed recurrent of the disease.

Conclusions: Our experience showed that endovascular treatment is not satisfactory for CAD recurrence even with atherectomy.

\section{Background}

Cystic adventitial disease (CAD) is a rare non-atherosclerotic condition in which fluid accumulates in the sub-adventitial layer, compressing the lumen of the arteries and veins. In $80-90 \%$ of cases, CAD is located in the popliteal artery, where it can cause intermittent claudication and critical limb ischaemia. 1,2 Although several techniques, including surgical and interventional methods, have been used to treat this disease, surgical resection and graft implantation were reported as the most reliable methods in many case reports. ${ }^{3}$ Treatment with percutaneous transluminal angioplasty (PTA) seems to be unsatisfactory owing to high recurrence rates. ${ }^{4}$ However, the use of an atherectomy device has not been previously attempted as a treatment method. Furthermore, rare cases of recurrence have been reported even after surgical treatment, but only a few reports have explored the appropriate treatment in the case of recurrence.

We here report a case in which rotational atherectomy was performed for the treatment of a patient with recurrent CAD who was initially treated with cyst excision and patch angioplasty surgery. A review of the literature indicated that this is the first case in which atherectomy was performed for the treatment of $C A D$ in general and particularly for recurrent CAD after surgery.

\section{Case Presentation}

A 67-year-old male patient presented to the emergency department with acute deterioration of walking ability caused by claudication in the left limb which had started on the previous day. On clinical examination, his left dorsalis pedis arterial pulse was weak but his sensory and motor functions were intact. He had a history of CAD of the left popliteal artery which was treated with excision of the cyst and patch angioplasty (3 months previously). Computed tomography angiography (CTA) imaging showed that the popliteal artery was almost completely occluded (Fig. 1). On the basis of the CTA findings, the cause of the obstruction was suspected to be a thrombotic occlusion. Therefore, we decided to perform 
angiography and additional interventions. Left lower-extremity arteriography was performed through an antegrade puncture of the left common femoral artery, which confirmed the narrowing and thrombotic occlusion of the popliteal artery (Fig. 2). In addition, a cystic lesion without a doppler signal was observed inside of the popliteal artery in colour Doppler ultrasound (US) performed during angiography, it was considered that the narrowing was likely accompanied by recurrence. Therefore, we performed rotational atherectomy as a treatment which can solve both the thrombotic occlusion and the recurrence. After placing the embolisation protection device (Spider FX; Covidien, Plymouth, MN) in the distal popliteal artery, we performed rotational atherectomy using Jetstream XC (Boston Scientific, Natick, MA). Additionally, balloon angioplasty was performed using a plain balloon (Passeo-18; BIOTRONIK AG, Buelach, Switzerland). Post-procedure angiography showed that atherectomy and balloon angioplasty resulted in a satisfactory increase in the luminal diameter. However, the patient's symptoms recurred 1 day after the rotational atherectomy and balloon angioplasty, and repeat CTA showed recurrent occlusion of the popliteal artery with intraluminal cystic lesion, suggesting recurrence of the disease. Despite the recurrence, the degree of claudication improved after the atherectomy procedure. Therefore, the patient was discharged with conservative treatment and intensive follow-up. During the follow-up, the degree of claudication worsened and surgical treatments were performed again. Left distal femoral artery to popliteal artery (below-the-knee) bypass with ipsilateral reversed great saphenous vein graft surgery was performed by an experienced vascular surgeon. Improvement in claudication was observed at the 6month postoperative follow-up, and the patient is continuing follow-up without additional treatment.

\section{Discussion And Conclusions}

CAD remains a rare cause of lower-limb ischaemia, with a prevalence of $0.1 \%$ among patients with intermittent claudication. ${ }^{5}$ The exact aetiology of CAD is not known, although four theories about its pathogenesis have been postulated: trauma theory, ganglion theory, systemic disorder theory, and developmental theory. ${ }^{6}$

Historically, surgical treatment of CAD is preferred over endovascular or minimally invasive techniques owing to the considerably lower recurrence rates after surgery. The main surgical treatments are resection of the affected popliteal arterial segment with interposition of an autologous vein graft and cyst enucleation. Original articles and textbooks recommend popliteal artery resection with graft interposition only in the presence of arterial occlusion or involvement of the media ${ }^{2,7}$; however, many vascular surgeons prefer this method even in cases in which the artery is not completely occluded. ${ }^{8}$

Percutaneous endovascular interventions, such as angioplasty and stenting, have been attempted, with unsatisfactory results. Endarterectomy and endovascular interventions do not directly address CAD, which originates from the adventitial layer of the artery. As a result, early recurrence is almost guaranteed to occur (as early as $8 \mathrm{~h}$ after treatment in some cases). ${ }^{2,8}$ Only one case of CAD recurrence successfully treated with balloon angioplasty has been reported, in which the patient had previously undergone surgical excision. ${ }^{9}$ Nevertheless, in our case, the decision to perform interventional treatment was based 
on the patient's history of percutaneous thrombectomy for the removal of a thrombus from the postoperative popliteal artery 2 months previously and on the misdiagnosis of thrombotic occlusion based on CTA findings. Angiography and Doppler US revealed an accompanying recurrence; however, it was difficult to perform surgery immediately and intravascular treatment was performed instead. We considered that the atherectomy device would destroy the cyst wall and consequently allow the drainage of internal fluids. However, the destruction of the cyst wall was insufficient even with atherectomy and balloon angioplasty, and CAD recurred the day after the procedure.

This is the first reported case in which atherectomy was performed for recurrent CAD. Our experience showed that endovascular treatment is not satisfactory for CAD recurrence, even with atherectomy. Although recurrence after the primary surgical treatment of CAD remains rare, similar symptoms may be observed once it occurs. The application of atherectomy was found to be unsatisfactory in this case. This report highlights the pitfalls which can be avoided by selecting surgical resection as a therapeutic approach.

\section{List Of Abbreviations}

CAD: Cystic adventitial disease; PTA: Percutaneous transluminal angioplasty; CTA: Computed tomography angiography; US: ultrasound.

\section{Declarations}

\section{Acknowledgements}

Not applicable.

\section{Authors' contributions}

JWY: drafted the manuscript, contributed to data and images collection; WC: conducted endovascular intervention, supervised the study and edited the manuscript; SMK: conducted surgery, participated in the design and coordination of the manuscript; $\mathrm{JH}$ : reviewed literature and provided consultation. All authors read and approved the final manuscript.

\section{Fundings}

This research was supported by the Chung-Ang University Research Grants in 2020.

\section{Availability of data and materials}

All data generated or analyzed during this study are included in this published article.

\section{Ethics approval and consent to participate}

Not applicable. 


\section{Consent for publication}

Written informed consent for publication of details was obtained from the patient.

\section{Competing interests}

The authors declared no potential competing interest with respect to the research, authorship, and/or publication of this article.

\section{References}

1. Desy NM and Spinner RJ. The etiology and management of cystic adventitial disease. Journal of vascular surgery 2014; 60: 235-245. e211.

2. Van Rutte $P$, Rouwet EV, Belgers $E$, et al. In treatment of popliteal artery cystic adventitial disease, primary bypass graft not always first choice: two case reports and a review of the literature. European Journal of Vascular and Endovascular Surgery 2011; 42: 347-354.

3. Paravastu SC, Regi JM, Turner DR, et al. A contemporary review of cystic adventitial disease. Vascular and endovascular surgery 2012; 46: 5-14.

4. Fox RL, Kahn M, Adler J, et al. Adventitial cystic disease of the popliteal artery: failure of percutaneous transluminal angioplasty as a therapeutic modality. Journal of vascular surgery 1985; 2: 464-467.

5. Mateo MMH, Hernando FJS, López IM, et al. Cystic adventitial degeneration of the popliteal artery: report on 3 cases and review of the literature. Annals of vascular surgery 2014; 28: 1062-1069.

6. Flanigan DP, Burnham SJ, Goodreau JJ, et al. Summary of cases of adventitial cystic disease of the popliteal artery. Annals of surgery 1979; 189: 165.

7. Galland B. Rutherford's Vascular Surgery, 7th edn. The Royal College of Surgeons of England, 2011.

8. Tsilimparis N, Hanack U, Yousefi S, et al. Cystic adventitial disease of the popliteal artery: an argument for the developmental theory. Journal of vascular surgery 2007; 45: 1249-1252.

9. Maged IM, Kron IL and Hagspiel KD. Recurrent cystic adventitial disease of the popliteal artery: successful treatment with percutaneous transluminal angioplasty. Vascular and endovascular surgery 2009; 43: 399-402.

\section{Figures}



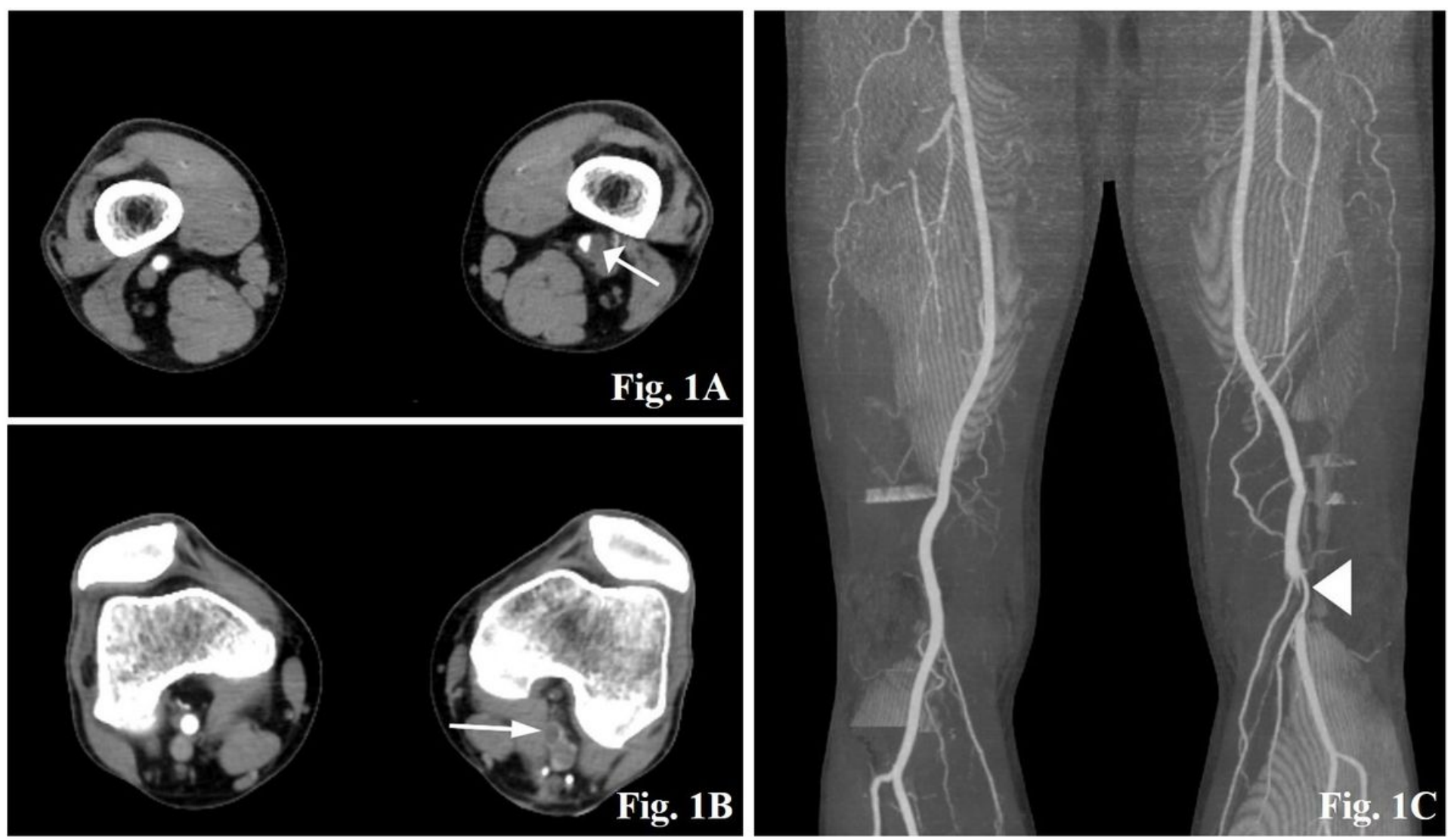

\section{Figure 1}

Computed tomography angiography (CTA) images of the lower extremity. (A) Preoperative initial axial image above the knee showing a cystic mass (white arrow) compressing the left popliteal artery. $(B, C)$ Images from the repeat CTA performed 3 months after the excision of the cyst with patch angioplasty, showing near-complete occlusion of popliteal artery at the level of the knee (B, white arrow). The right popliteal artery appears normal. Coronal maximal intensive projection images (C) show short-segment non-opacification of the left popliteal artery at the level of the knee (white arrowheads). 


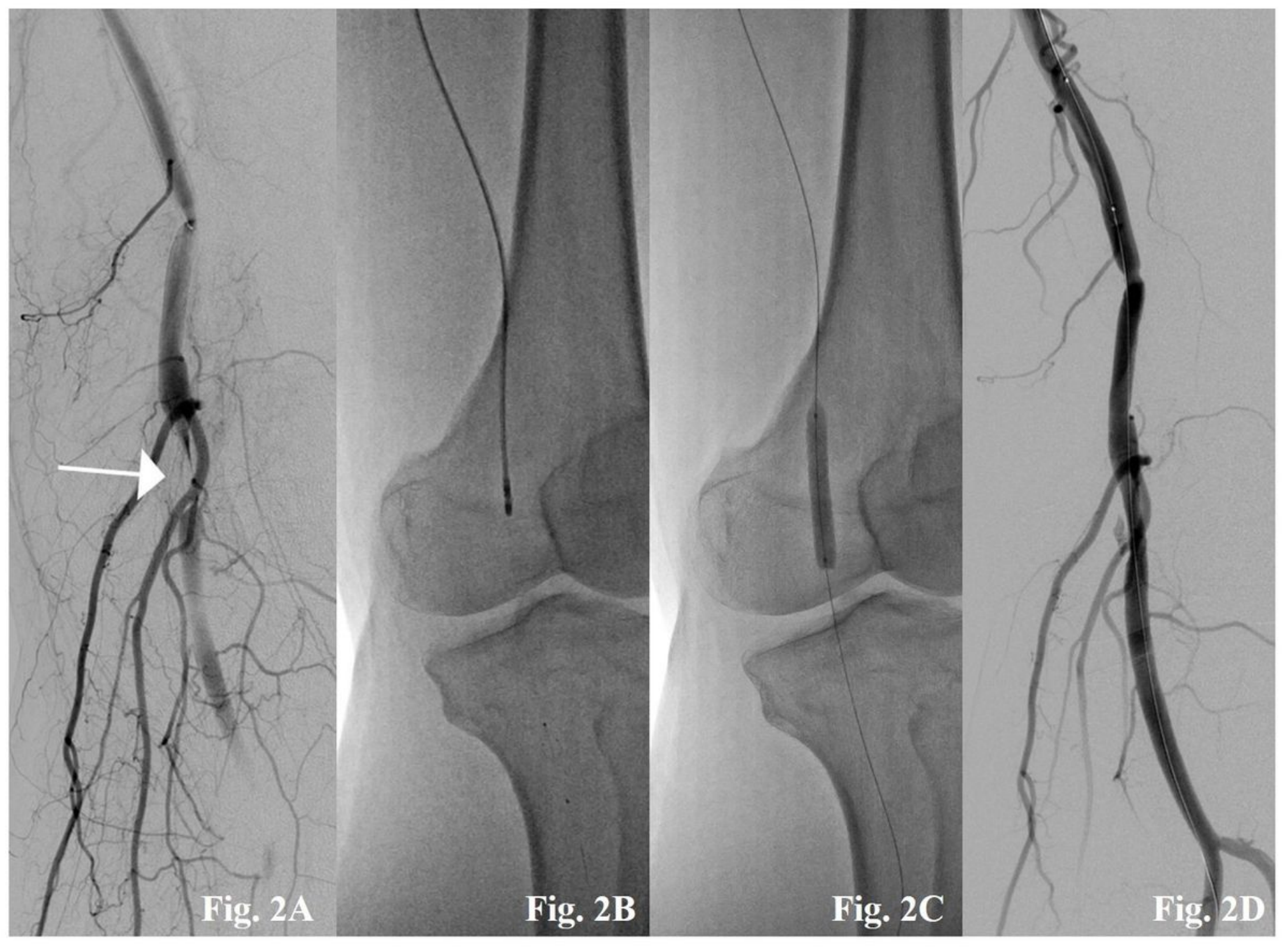

\section{Figure 2}

(A) Left lower-extremity arteriogram with digital subtraction showing focal extrinsic narrowing and nearcomplete occlusion (arrow) of the popliteal artery (arrow). (B) Spot film image at the level of the knee showing rotational atherectomy of the lesion. (C) Additional plain balloon angioplasty. (D) Control angiogram showing the recanalised patent popliteal artery lumen after atherectomy and percutaneous transluminal angioplasty. 